\title{
Relative indexes of cutaneous blood perfusion measured by real-time laser Doppler imaging (LDI) in healthy volunteers
}

\author{
S. Morteza Seyed Jafari ${ }^{\mathrm{a}}$, Megir Schawkat ${ }^{\mathrm{a}}$, Dimitri Van De Ville ${ }^{\mathrm{b}, \mathrm{c}}$, Maziar Shafighi ${ }^{\mathrm{a}, *}$ \\ a Department of Plastic, Reconstructive and Hand Surgery, University Hospital, Inselspital, University of Berne, Switzerland \\ b Department of Radiology and Medical Informatics, University of Geneva, Switzerland \\ c Institute of Bioengineering, Ecole Polytechnique Fédérale de Lausanne, Switzerland
}

\section{A R T I C L E I N F O}

Article history:

Accepted 18 April 2014

Available online 29 April 2014

\section{Keywords:}

Laser Doppler imaging

Microcirculation

Blood perfusion

\begin{abstract}
A B S T R A C T
We used real-time LDI to study regional variations in microcirculatory perfusion in healthy candidates to establish a new methodology for global perfusion body mapping that is based on intra-individual perfusion index ratios. Our study included 74 ( 37 female) healthy volunteers aged between 22 and 30 years (mean 24.49). Imaging was performed using a recent microcirculation-imaging camera (EasyLDI) for different body regions of each volunteer. The perfusion values were reported in Arbitrary Perfusion Units (APU). The relative perfusion indexes for each candidate's body region were then obtained by normalization with the perfusion value of the forehead. Basic parameters such as weight, height, and blood pressure were also measured and analyzed. The highest mean perfusion value was reported in the forehead area (259.21 APU). Mean perfusion in the measured parts of the body correlated positively with mean forehead value, while there was no significant correlation between forehead blood perfusion values and room temperature, BMI, systolic blood pressure and diastolic blood pressure $(p=0.420,0.623,0.488,0.099$, respectively). Analysis of the data showed that perfusion indexes were not significantly different between male and female volunteers except for the ventral upper arm area $(p=.001)$. LDI is a non-invasive, fast technique that opens several avenues for clinical applications. The mean perfusion indexes are useful in clinical practice for monitoring patients before and after surgical interventions. Perfusion values can be predicted for different body parts for patients only by taking the forehead perfusion value and using the perfusion index ratios to obtain expected normative perfusion values.
\end{abstract}

(c) 2014 Elsevier Inc. All rights reserved.

\section{Introduction}

Microcirculation is the main link between the cardiovascular system and cellular interfaces (Gargiulo et al., 2013), and exchange of material between the intravascular and interstitial spaces occurs mainly at this level, which is essential for tissue viability. This fact advocates the need to evaluate microvascular circulation in clinical practice (Tulevski et al., 1999). The detection of microvascular dysfunction is potentially necessary for the early diagnosis of microvascular disorders and the development of therapeutical concepts for their treatment (Keymel et al., 2010). It has been suggested that the cutaneous microcirculation may reflect generalized systemic vascular dysfunction in magnitude and underlying mechanisms (Cracowski et al., 2006; Holowatz et al., 2008; Mahé et al., 2012). Assessment of cutaneous microvascular function can be performed by both invasive and non-invasive techniques (Mahé et al., 2012). Among non-invasive perfusion measurement techniques, laser Doppler imaging (LDI) gives access to twodimensional measurements of the tissue perfusion (Nilsson, 1997)

\footnotetext{
* Corresponding author at: Universitätsklinik für Plastische und Handchirurgie Bern, Inselspital Bern, 3010 Bern, Switzerland.

E-mail address: maziar.shafighi@insel.ch (M. Shafighi).
}

and offers an applicable alternative to evaluate real-time microcirculation changes in the skin (Gargiulo et al., 2013; Nilsson, 1997). This technique eliminates the need of a fixed probe to the skin and allows measurement from a distance of a larger skin area (Svedman et al., 1998; Wårdell et al., 1993). Interestingly, by taking continuous measurements from each area (i.e., 130 images in $10 \mathrm{~s}$ ) and reporting the temporally averaged value for the measured perfusion, EasyLDI can overcome substantial temporal variability of baseline blood flow measurements in the same subject (Jan et al., 2005). LDI perfusion measurements of different parts of the body have several applications, therefore attracting the interest of various research and clinical groups (Hagblad et al., 2010), however, there are no unique standardized perfusion values for each part of the body in the literature. Although vascular anatomy may show differences among individuals and even intraindividually, such as changes due to development, aging and regional differences, the basic vascular "blueprint" is the same. Knowledge about the potential vascular anatomical territory of the skin, especially if it can be obtained before surgery, is helpful to the design of safe skin flap transplantation in reconstructive surgery (Leutenegger et al., 2011; Taylor et al., 2011). Therefore, in order to have applicable standardized values for different regions of the body, we applied real-time LDI to study regional variations in skin microcirculatory perfusion in a 


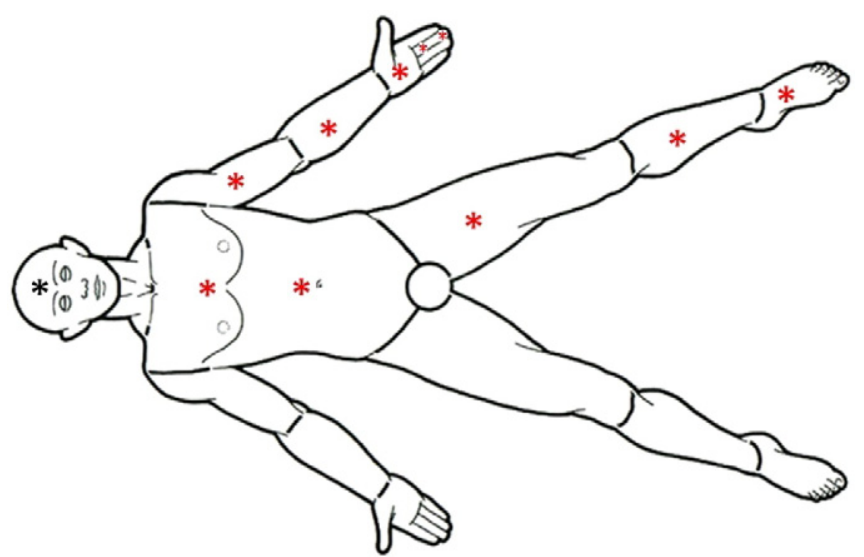

Fig. 1. Blood perfusion in the body regions. The black marker shows the forehead area, and the red ones show the area for which the perfusion measurements were done dorsally and ventrally (Harbi and Thacher, 2013).

group of healthy candidates as to provide a new methodology for global perfusion body mapping based on intra-individual perfusion ratios.

\section{Materials and methods}

\section{Patient selection}

Our study included 74 healthy medical students ( 37 female). Exclusion criteria included smoking, known microcirculatory disorders, comorbidities influencing the acquisition of clinical data (hypertension, rheumatologic disorder, asthma, diabetes, trauma), or unstable medical condition affecting imaging. All procedures of this study were in accordance with the ethical standards of the Ethical Committee of the Canton of Bern (KEK) on human experimentation (No. 157/12) and with the Helsinki Declaration of 1975, as revised in 1983. Each volunteer was also required to sign the informed consent and received a code number so that data could be anonymized.

\section{Device}

Laser Doppler imaging was performed using a commerciallyavailable microcirculation-imaging camera (EasyLDI; Aimago SA, Lausanne, Switzerland). The field-of-view (FOV) of the LDI camera is $7 \times 7 \mathrm{~cm}^{2}$ with $150 \times 150 \mu \mathrm{m}^{2}$ pixel size. The frame rate of the perfusion images is $13 \mathrm{~Hz}$. The instrument also provides simultaneously a regular color image of the FOV. An LDI instrument consists of a light source (a monochromatic laser with a long wavelength), a fast detector, and a unit for processing and recording the detected signals. When the laser illuminates the tissue, part of the light is shifted in wavelength by interaction with moving red blood cells due to the Doppler effect. After detection of the coherently mixed static and dynamic light fields, the interference of both fields makes a detectable beating of the light intensity that is recorded by the device (Humeau-Heurtier et al., 2013; Leutenegger et al., 2011).

\section{Measurement protocol}

On the day of the imaging acquisition, volunteers' weight and height were taken to calculate BMI and their birth dates were noted. These measurements were taken between 8 and 10 a.m. in a quiet room with temperatures ranging from 19.6 to $27.6{ }^{\circ} \mathrm{C}$. Confirmation that the patient had neither eaten nor drank coffee and tea in the morning. The volunteer was asked to disrobe to his or her underwear, lie on their back on the table and find a comfortable position. Five minutes later, the arterial blood pressure was obtained. Then, the candidate had been resting comfortably for $10 \mathrm{~min}$, and then blood perfusion imaging began in supine positions from 11 body regions (Fig. 1). Afterwards, imaging from 10 body areas in prone position was done, $5 \mathrm{~min}$ later. At each of these locations, a 10-s video of perfusion was saved along with the corresponding color photograph for future location identification. This resulted in 130 absolute perfusion measurements for each site. For each video sequence, a region of interest (ROI) was manually defined and applied to the same location for all images in the video sequence. Next, the average perfusion unit per unit area of the ROI was plotted versus time. The recordings for each region included given in Arbitrary Perfusion Units (APU) (Figs. 2, 3). Data were grouped by body region for all volunteers.

\section{Image analysis}

Images were grouped by body location for all candidates, and transferred to the EasyLDI Studio software, where the average perfusion per unit per area of ROI was then recorded for each body region of each volunteer (Harbi and Thacher, 2013). Since vascular anatomy may be different among individuals and even between opposite sides in the same individual individuals (Leutenegger et al., 2011; Taylor et al., 2011), it is necessary to omit the possible intra-individual varieties to have opportunity to compare this data between the candidates. So, based on our assumption that the forehead had the highest absolute perfusion value in each volunteer, we used the absolute perfusion of each volunteers' forehead as the reference number for each person (i.e., corresponding to perfusion index of 1 ), then index values of the

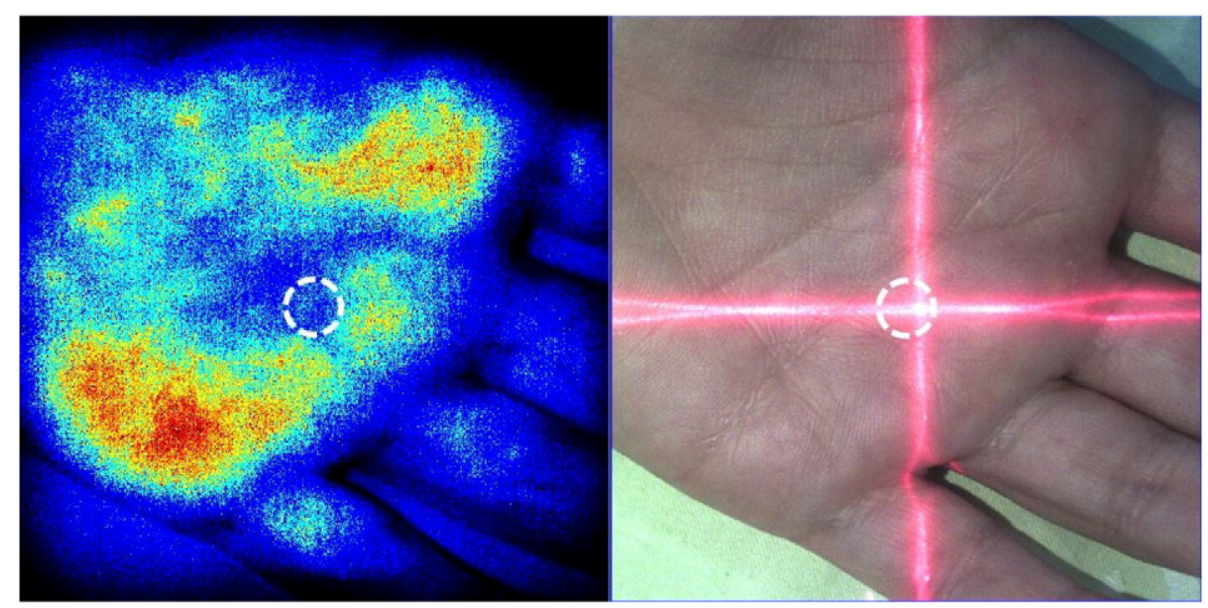

Fig. 2. The left image is a LDI image, and the right image shows the photo of the same area. A region of interest (ROI) can be seen as a circle in both images. 


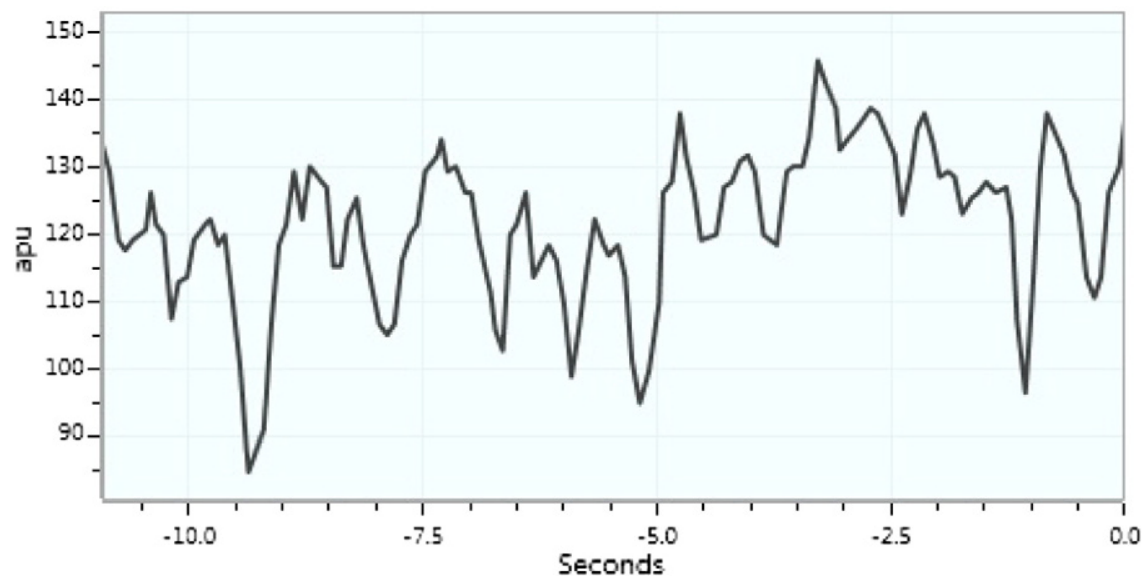

Fig. 3. This plat shows the time series of the center ROI at the time of snapshot.

other body parts were calculated by dividing that measured value by the perfusion value of the forehead.

\section{Statistical analysis}

Data were grouped by body location for all volunteers. Standardized effect sizes were computed from the LDI mean perfusion values in APU in different body regions. In order to validate the relationship between perfusion measurements of the forehead and other body regions, Pearson normalized correlation coefficient was computed across subjects. Two-sample t-test was also applied to compare the differences of perfusion indexes between male and female volunteers. In all cases, significant results were considered for $p$-values less than 0.05 . Due to multiple comparisons we also corrected the $p$-values for the correlation coefficients, with the number of other body regions (i.e., the corrected $\alpha$-level corresponded to 0.0025 ).

\section{Results}

Our study included 74 healthy medical students (37 female, 37 male), aged between 22 and 30 years (mean 24.49). The candidates had mean BMI of $23.45 \mathrm{~kg} / \mathrm{m}^{2}$ and mean systolic and diastolic blood pressures of $119.23 \mathrm{~mm} \mathrm{Hg}$, and $69.16 \mathrm{~mm} \mathrm{Hg}$, respectively (Table 1).

\section{LDI mean perfusion values in APU in different body regions}

In our LDI perfusion measurements among all body regions, forehead perfusion measurements ranged between 102.67 and 519.39 APU and had the highest mean values in APU (mean 259.21), while sole of the foot measurements ranged between 22.10 and 224.28 APU corresponding the lowest mean value in APU (mean 88.63). Moreover, standardized effect sizes for different body regions were calculated as the ratio between the mean and SE of the perfusion values in APU. The forehead region ranked second in terms of standardized effect size (Table 2), but there was no significant difference with the first ranked region (dorsal upper arm).

Table 1

General descriptive table.

\begin{tabular}{lccc}
\hline & Range & Mean & SE \\
\hline Age (years) & $22-30$ & 24.49 & .214 \\
Room temperature $\left({ }^{\circ} \mathrm{C}\right)$ & $19.6-27.6$ & 23.901 & .2137 \\
Body mass index $\left(\mathrm{kg} / \mathrm{m}^{2}\right)$ & $17.22-30.00$ & 23.45 & .47980 \\
Systolic BP $(\mathrm{mm} \mathrm{Hg})$ & $92-160$ & 119.23 & 1.322 \\
Diastolic BP $(\mathrm{mm} \mathrm{Hg})$ & $45-90$ & 69.16 & .857 \\
\hline
\end{tabular}

Correlation of forehead perfusion value with other measured values

The Pearson correlation test showed that there is no significant correlation between forehead perfusion values and room temperature $(p, r=.420, .095)$. Neither correlation between forehead perfusion values and systolic blood pressure $(p, r=.488,-.082)$ and diastolic blood pressure was significant $(p, r=.099, .193)$. Forehead perfusion values have also no correlation with BMI $(p, r=.623,-.058)$ (Table 3 and Fig. 4). Interestingly, we found significant positive correlations between the measured perfusion values of the forehead and all other body regions for both genders, and all candidates $(p=.000)$ (Table 4 and Fig. 5).

\section{Relative index values of perfusion}

We reported the mean index values \pm SD reported for different body regions. The gender specific mean index values \pm SD for each body part are also presented in Table 5 . Comparison of the perfusion

Table 2

LDI mean perfusion values in APU in different body regions.

\begin{tabular}{|c|c|c|c|c|}
\hline & Range & Mean & SE & $\begin{array}{l}\text { Standardized } \\
\text { effect size }\end{array}$ \\
\hline Forehead value (APU) & 102.67-519.39 & 259.21 & 8.53 & 30.39 \\
\hline Chest value (APU) & $51.15-363.02$ & 169.29 & 6.54 & 25.89 \\
\hline Upper back value (APU) & $74.03-478.47$ & 221.46 & 8.05 & 27.51 \\
\hline Abdomen value (APU) & $61.90-484.21$ & 206.89 & 7.74 & 26.73 \\
\hline Lower back value (APU) & 58.59-396.66 & 189.69 & 6.63 & 28.61 \\
\hline Ventral upper arm value (APU) & $35.29-296.15$ & 142.75 & 5.60 & 25.49 \\
\hline Dorsal upper arm value (APU) & $51.53-352.34$ & 171.38 & 5.55 & 30.88 \\
\hline Ventral forearm value (APU) & $33.78-293.52$ & 124.69 & 5.47 & 22.80 \\
\hline Dorsal forearm value (APU) & $31.65-231.76$ & 115.23 & 4.53 & 25.44 \\
\hline Ventral hand value (APU) & $66.34-465.24$ & 206.76 & 7.68 & 26.92 \\
\hline Dorsal hand value (APU) & $26.31-222.19$ & 104.69 & 3.85 & 27.19 \\
\hline $\begin{array}{l}\text { Ventral proximal middle finger } \\
\text { value (APU) }\end{array}$ & $70.28-429.25$ & 221.38 & 7.46 & 29.68 \\
\hline $\begin{array}{l}\text { Dorsal proximal middle finger } \\
\text { value (APU) }\end{array}$ & $30.28-255.58$ & 105.22 & 4.50 & 23.38 \\
\hline $\begin{array}{l}\text { Ventral distal middle finger } \\
\text { value (APU) }\end{array}$ & $39.41-333.50$ & 158.77 & 5.78 & 27.47 \\
\hline $\begin{array}{l}\text { Dorsal distal middle finger } \\
\text { value (APU) }\end{array}$ & $31.95-265.24$ & 117.69 & 4.48 & 26.27 \\
\hline Ventral thigh value (APU) & $48.65-369.43$ & 157.34 & 6.02 & 26.14 \\
\hline Dorsal thigh value (APU) & $46.23-383.96$ & 160.23 & 5.77 & 27.77 \\
\hline Ventral lower leg value (APU) & $41.65-290.48$ & 134.02 & 5.18 & 25.87 \\
\hline Dorsal lower leg value (APU) & $47.04-265.04$ & 132.76 & 4.71 & 28.19 \\
\hline Ventral foot value (APU) & $22.65-200.83$ & 96.77 & 3.81 & 25.40 \\
\hline Foot sole value (APU) & $22.10-224.28$ & 88.63 & 3.98 & 22.27 \\
\hline
\end{tabular}


Table 3

Correlation of forehead perfusion value with temperature, BMI and blood pressure.

\begin{tabular}{|c|c|c|c|c|c|c|c|c|}
\hline \multicolumn{9}{|l|}{ Pearson correlation test } \\
\hline & \multicolumn{2}{|c|}{ Room temperature } & \multicolumn{2}{|c|}{ Body mass index } & \multicolumn{2}{|c|}{ Systolic blood pressure } & \multicolumn{2}{|c|}{ Diastolic blood pressure } \\
\hline & $r$ & Sig. (2-tailed) & $r$ & Sig. (2-tailed) & $r$ & Sig. (2-tailed) & $r$ & Sig. (2-tailed) \\
\hline Forehead value (APU) & .095 & .420 & -.058 & .623 & -.082 & .488 & .193 & .099 \\
\hline
\end{tabular}

indexes between male and female candidates showed significant perfusion differences in the ventral upper arm area $(p=.001)$ while there is no significant difference to other body regions (Table 5 ).

\section{Discussion}

Although there are many techniques suitable for measuring microvascular perfusion of body parts, no single technology has found wide applicability (Smit et al., 2010). However, recent reviews have suggested that Doppler probes offer as the best one (Abdel-Galil and Mitchell, 2009). Laser doppler perfusion imaging (Bonner and Nossal, 1981; Draijer et al., 2009; Serov et al., 2005) and related techniques such as laser speckle contrast analysis and laser speckle imaging (Briers, 2001, 2007; Draijer et al., 2009) are established techniques for determining skin perfusion maps, burn wound assessment (Droog et al., 2001; Hoeksema et al., 2009; Kloppenberg et al., 2001), cerebral blood flow study in animals (Draijer et al., 2009), and drug uptake studies (e.g., using iontophoresis) (Turner et al., 2008). Some of the issues are that these devices' probes require direct contact with the skin, measurements are not highly reproducible, and the sampled region is very small (1-2 mm). Laser Doppler imaging (LDI) technique is based on extending the principle of laser Doppler flowcytometry (LDF) which allows measurement from a distance of a larger skin area (Binzoni and Van De Ville, 2008; Svedman et al., 1998; Wårdell et al., 1993).

We used EasyLDI device to obtain perfusion values from predetermined skin area in young healthy volunteers. Data were grouped per subject and body region. Our study showed that the forehead had the highest mean APU value among all body regions (i.e., APU of 259.21). Though, local changes of the room temperature can induce regional effects, (Cooke et al., 1990) there was no significant correlation between forehead blood perfusion and room temperature $(p=$ .420). Millet et al. (2011) reported gradual increase in skin perfusion when performing local heating at $36,39,42$, and $44{ }^{\circ} \mathrm{C}$ on the forearm of healthy volunteers and measuring cutaneous blood flux with LDI. However, there is a core temperature threshold to start vasodilation, which leads to the delay in the initiation of active vasodilation and increase in skin blood flow (González-Alonso et al., 2008; Simmons

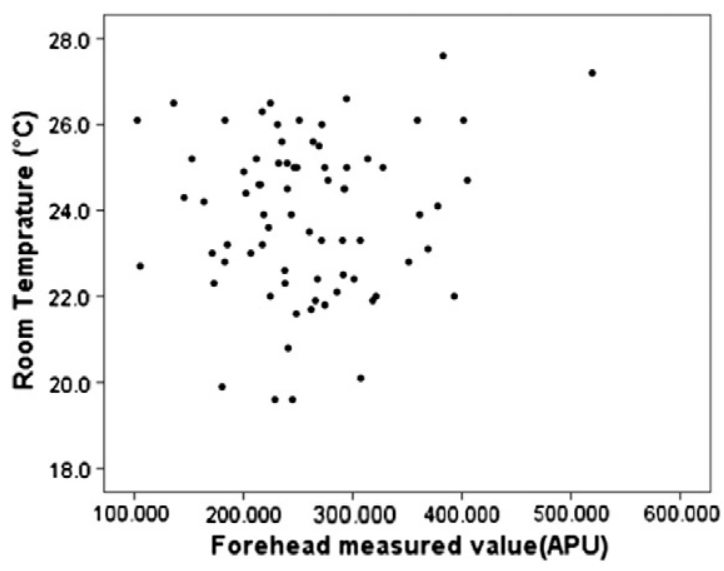

Fig. 4. Correlation of perfusion in the forehead and room temperature. et al., 2011). So, the present differences could be due to the narrow range of room temperature in our study and absence of any direct heating to reach temperature to the necessary threshold for the skin blood flow significant increase.

There was also no significant correlation between forehead perfusion values and BMI, systolic blood pressure and diastolic blood pressure ( $p=.623, .488, .099)$, but interestingly the changes of perfusion across subjects correlated significantly between the forehead and other body regions. These positive correlations could be related to unity and connectivity in cardiovascular system that regulates the perfusions in the body. Since the anatomy of the cutaneous circulation shows a great variability due to development and aging, regional differences, effects of even minor pathology (Leutenegger et al., 2011), complex structure of tissue, and the complexity of laser-tissue interactions, it is not easy to work with absolute APU values for comparison. As a result, a relative measurement of perfusion has been recommended (Forrester et al., 2002). Harbi and Thacher in their blood perfusion body mapping study with a LDI device, set forehead value as the reference for normalizing the data (Harbi and Thacher, 2013). In the current study, highly significant positive correlations between perfusion in the forehead and other body regions were convincing to use this value as the reference also for our study. We then defined the perfusion index for each body region as relative perfusion with respect to the forehead. This relative perfusion measurement gave us the opportunity to compare this data between the volunteers. In our study on the 74 candidates the ventral proximal middle finger and upper back (mean index $=.859 \pm .120$, $.853 \pm .099$ ) presented the highest and the sole of the foot, and ventral foot (mean index $=.346 \pm .100, .375 \pm .077$ ) had the lowest mean indexes among the body regions, while Harbi and Thacher reported that the head ( $74 \pm 11)$ had the highest and the foot $(29 \pm 9)$ had the lowest mean (Harbi and Thacher, 2013). In a study on cutaneous microcirculation Raju et al. stated that the capillary density/filling is mean $86 \pm 19$ at the ankle gradually declining till the mid-abdomen and then increasing thereafter at the xiphisternum. There is a gradual increase in the upper chest skin, rising even more in the forehead which has a greater capillary density fill than the ankle (Raju et al., 2012). The results of the study by Tur E et al. revealed that a collection of regions (fingers, palms, face, ears) has much higher cutaneous perfusion than all other positions (Tur et al., 1983). This low perfusion in the foot area could be due to having most peripheral position and weaker vascularity in comparison to other body parts. The comparison of indexes between male and female participants in the study showed that the indexes in the female were lower than male volunteers', but this differences in perfusions were not statistically significant, except in the ventral upper arm area $(p=.001)$. Although it is believed that the cutaneous blood flow in women is less than that in men due to differences in central, rather than local, control mechanisms (Cooke et al., 1990), our semi-quantitative relative measurement of the data may eliminate this gender effects on the perfusion indexes.

LDI allows assessing tissue perfusion and cutaneous microvascular function in a non-contact, non-invasive, and quantitative way (Cracowski et al., 2006; Forrester et al., 2002; Hoeksema et al., 2009).

Among the body regions considered in this study, we found that the forehead showed the highest skin mean perfusion value and was among the top regions in terms of standardized effect size. Therefore, we considered the forehead region as the reference for defining the relative 
Table 4

Correlation of forehead perfusion value with other measured areas. ( ${ }^{*}$ Sig. (2-tailed) $=.000$ for all correlation analysis).

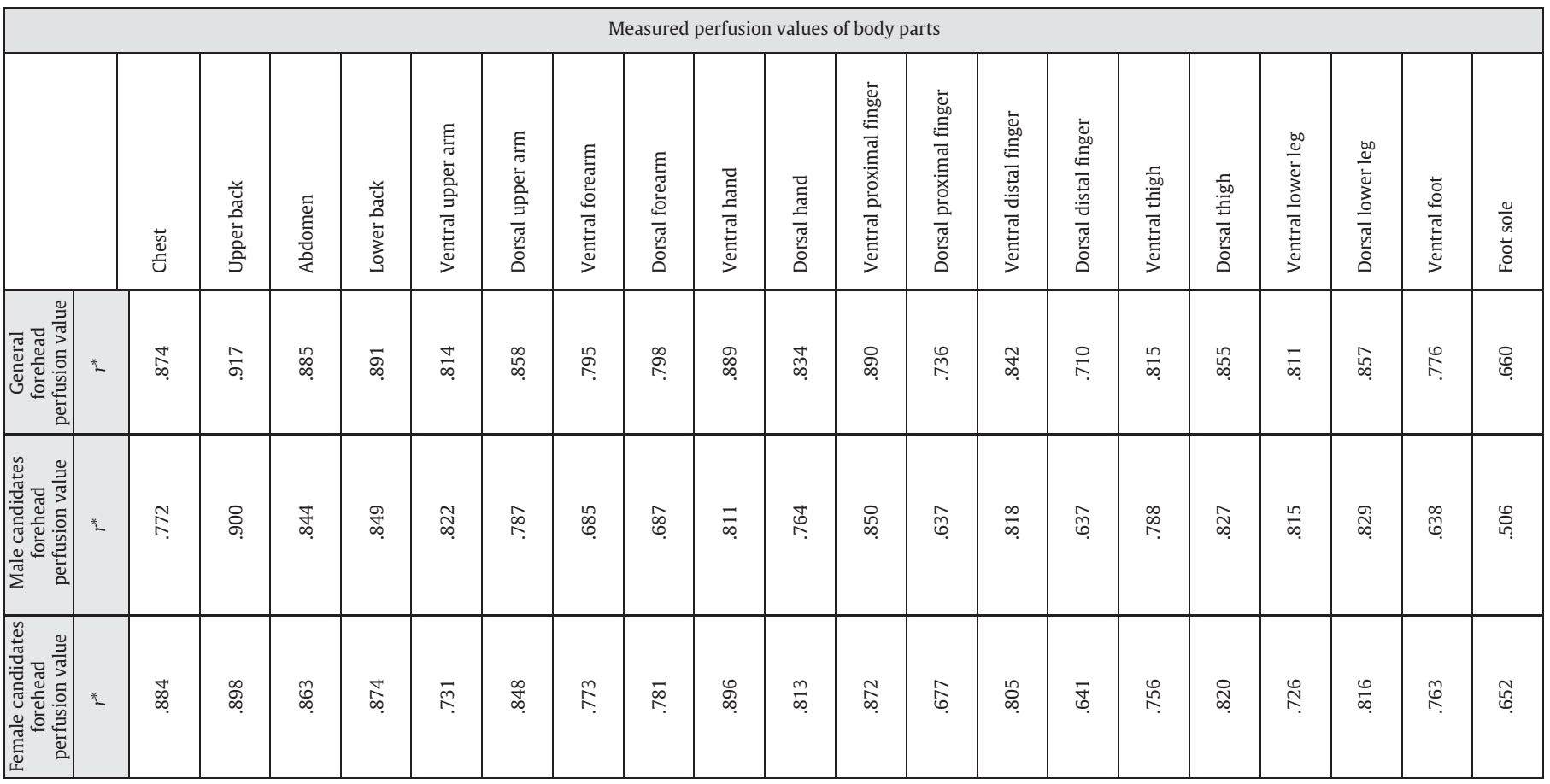

index values. An interesting avenue for further research could be to investigate specifically the choice of the reference region on the robustness of the index values.

In order to overcome the main (temporal) variability of the baseline blood flow, we took continuously 130 measurements during $10 \mathrm{~s}$ from each area and reported the temporally averaged values. However, it should be noted that follow-up studies are needed to compare these results with other perfusion measurement techniques.

The full-field LDI of dermal blood flow has gained much interest in recent years, because abnormally altered peripheral blood flow provides a practical indicator for many common diseases such as peripheral vascular disease, diabetic conditions, skin irritants, burns, and organ transplants (Leutenegger et al., 2011; Nilsson, 1997; Serov et al., 2005).

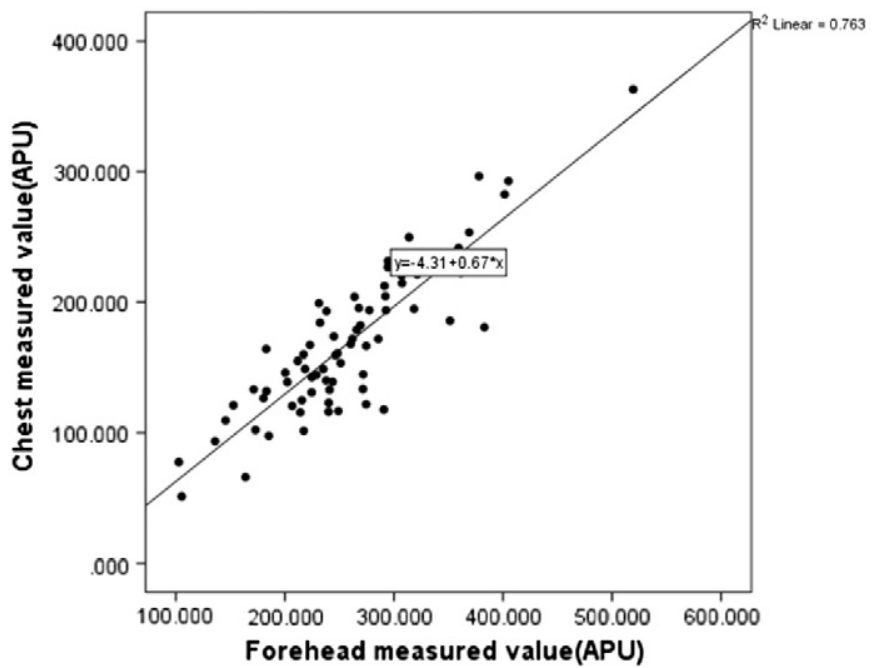

Fig. 5. Correlation of perfusion in the forehead and chest.
LDI has a high potential for applications in reconstructive surgery including objective assessment of burn depth, of the vascular adequacy of recipient beds for the placement of skin grafts, and of the response of hypertrophic scarring to therapeutic interventions (Arnold et al., 1995), as well as to monitor the patients before and after surgical interventions. For this purpose the mean perfusion indexes should be taken

Table 5

Index table (general and gender specific).

\begin{tabular}{|c|c|c|c|c|}
\hline \multicolumn{5}{|l|}{ Index table } \\
\hline \multirow[t]{2}{*}{ Area } & \multirow{2}{*}{$\begin{array}{l}\text { General } \\
\text { mean } \\
\text { index } \pm \mathrm{SD}\end{array}$} & \multicolumn{3}{|c|}{ Gender specific mean index } \\
\hline & & $\begin{array}{l}\text { Male } \\
(\text { Mean } \pm \text { SD })\end{array}$ & $\begin{array}{l}\text { Female } \\
(\text { Mean } \pm \text { SD })\end{array}$ & $\begin{array}{l}\text { Sig. (2-tailed) } \\
\text { (Between } \\
\text { genders) }\end{array}$ \\
\hline Chest index & $.65 \pm .11$ & $.62 \pm .12$ & $.68 \pm .09$ & .024 \\
\hline Upper back index & $.85 \pm .10$ & $.83 \pm .10$ & $.87 \pm .10$ & .054 \\
\hline Abdomen index & $.80 \pm .11$ & $.77 \pm .11$ & $.83 \pm .11$ & .027 \\
\hline Lower back index & $.73 \pm .10$ & $.71 \pm .10$ & $.76 \pm .09$ & .056 \\
\hline Ventral upper arm index & $.55 \pm .10$ & $.51 \pm .09$ & $.59 \pm .11$ & .001 \\
\hline Dorsal upper arm index & $.67 \pm .10$ & $.67 \pm .11$ & $.67 \pm .10$ & .828 \\
\hline Ventral forearm Index & $.48 \pm .11$ & $.44 \pm .10$ & $.51 \pm .10$ & .004 \\
\hline Dorsal forearm index & $.45 \pm .09$ & $.43 \pm .09$ & $.47 \pm .08$ & .058 \\
\hline Ventral hand index & $.80 \pm .12$ & $.79 \pm .13$ & $.81 \pm .10$ & .597 \\
\hline Dorsal hand index & $.41 \pm .07$ & $.39 \pm .08$ & $.42 \pm .07$ & .086 \\
\hline $\begin{array}{l}\text { Ventral proximal middle } \\
\text { finger index }\end{array}$ & $.86 \pm .12$ & $.85 \pm .12$ & $.87 \pm .12$ & .452 \\
\hline $\begin{array}{l}\text { Dorsal proximal middle } \\
\text { finger index }\end{array}$ & $.41 \pm .10$ & $.38 \pm .09$ & $.44 \pm .10$ & .007 \\
\hline $\begin{array}{l}\text { Ventral distal middle } \\
\text { finger index }\end{array}$ & $.62 \pm .10$ & $.61 \pm .11$ & $.62 \pm .10$ & .939 \\
\hline $\begin{array}{l}\text { Dorsal distal middle } \\
\text { finger index }\end{array}$ & $.46 \pm .11$ & $.45 \pm .11$ & $.47 \pm .11$ & .384 \\
\hline Ventral thigh index & $.61 \pm .11$ & $.58 \pm .10$ & $.64 \pm .12$ & .044 \\
\hline Dorsal thigh index & $.62 \pm .10$ & $.61 \pm .10$ & $.63 \pm .09$ & .300 \\
\hline Ventral lower leg index & $.52 \pm .1$ & $.50 \pm .09$ & $.54 \pm .11$ & .059 \\
\hline Dorsal lower leg index & $.51 \pm .08$ & $.51 \pm .08$ & $.52 \pm .08$ & .377 \\
\hline Ventral foot index & $.37 \pm .08$ & $.36 \pm .08$ & $.39 \pm .07$ & .092 \\
\hline Foot sole index & $.35 \pm .10$ & $.34 \pm .11$ & $.35 \pm .09$ & .720 \\
\hline
\end{tabular}


into consideration in the clinic for skin perfusion monitoring of the patients before and after the operations such as reconstructive microvascular surgeries, revascularization or replantation of extremities, replantation etc. In particular, surgeons could predict the baseline cutaneous perfusion values of different body parts for the patients by taking the skin perfusion values from the forehead with easy LDI and applying the index to obtain normative values in different body regions. It should be mentioned that in the clinical applications of this numbers the body regions and ROI should be selected the same as our study, so more studies are needed to evaluate the clinical effectiveness of proposed indexes.

\section{Conflict of interest}

None.

\section{Acknowledgments}

This work was also supported in part by the KTI/CTI (under grant 12976.2).

\section{References}

Abdel-Galil, K., Mitchell, D., 2009. Postoperative monitoring of microsurgical free tissue transfers for head and neck reconstruction: a systematic review of current techniques-part I. Non-invasive techniques. Br. J. Oral Maxillofac. Surg. 47, 351-355.

Arnold, F., et al., 1995. Perfusion imaging of skin island flap blood flow by a scanning laser-Doppler technique. Br. J. Plast. Surg. 48, 280-287.

Binzoni, T., Van De Ville, D., 2008. Full-field laser-Doppler imaging and its physiological significance for tissue blood perfusion. Phys. Med. Biol. 53, 6673.

Bonner, R., Nossal, R., 1981. Model for laser Doppler measurements of blood flow in tissue. Appl. Opt. 20, 2097-2107.

Briers, J.D., 2001. Laser Doppler, speckle and related techniques for blood perfusion mapping and imaging. Physiol. Meas. 22, R35.

Briers, J.D., 2007. Laser speckle contrast imaging for measuring blood flow. Opt. Appl. 37, 139.

Cooke, J.P., et al., 1990. Sex differences in control of cutaneous blood flow. Circulation 82, 1607-1615.

Cracowski, J.-L., et al., 2006. Methodological issues in the assessment of skin microvascular endothelial function in humans. Trends Pharmacol. Sci. 27, 503-508.

Draijer, M., et al., 2009. Twente optical perfusion camera: system overview and performance for video rate laser Doppler perfusion imaging. Opt. Express 17, 3211-3225.

Droog, E., et al., 2001. Measurement of depth of burns by laser Doppler perfusion imaging. Burns 27, 561-568.

Forrester, K., et al., 2002. Comparison of laser speckle and laser Doppler perfusion imaging: measurement in human skin and rabbit articular tissue. Med. Biol. Eng. Comput. 40, 687-697.
Gargiulo, S., et al., 2013. Effects of some anesthetic agents on skin microcirculation evaluated by laser Doppler perfusion imaging in mice. BMC Vet. Res. 9, 255.

González-Alonso, J., et al., 2008. The cardiovascular challenge of exercising in the heat J. Physiol. 586, 45-53.

Hagblad, J., et al., 2010. A technique based on laser Doppler flowmetry and photoplethysmography for simultaneously monitoring blood flow at different tissue depths. Med. Biol. Eng. Comput. 48, 415-422.

Harbi, P., Thacher, T., 2013. Body mapping of human cutaneous microcirculatory perfusion using a real-time laser Doppler imager. Diab. Vasc. Dis. Res. 10, 187-190.

Hoeksema, H., et al., 2009. Accuracy of early burn depth assessment by laser Doppler imaging on different days post burn. Burns 35, 36-45.

Holowatz, L.A., et al., 2008. The human cutaneous circulation as a model of generalized microvascular function. J. Appl. Physiol. 105, 370-372.

Humeau-Heurtier, A., et al., 2013. Skin perfusion evaluation between laser speckle contrast imaging and laser Doppler flowmetry. Opt. Commun. 291, 482-487.

Jan, Y.-K., et al., 2005. Analysis of week-to-week variability in skin blood flow measurements using wavelet transforms. Clin. Physiol. Funct. Imaging 25, 253-262.

Keymel, S., et al., 2010. Characterization of the non-invasive assessment of the cutaneous microcirculation by laser Doppler perfusion scanner. Microcirculation 17, 358-366.

Kloppenberg, F., et al., 2001. Perfusion of burn wounds assessed by laser Doppler imaging is related to burn depth and healing time. Burns 27, 359-363.

Leutenegger, M., et al., 2011. Real-time full field laser Doppler imaging. Biomed. Opt. Express 2, 1470.

Mahé, G., et al., 2012. Assessment of skin microvascular function and dysfunction with laser speckle contrast imaging. Circ. Cardiovasc. Imaging 5, 155-163.

Millet, C., et al., 2011. Comparison between laser speckle contrast imaging and laser Doppler imaging to assess skin blood flow in humans. Microvasc. Res. 82, 147-151.

Nilsson, G., 1997. Laser Doppler perfusion imaging. Engineering in Medicine and Biology Society, 1996. Bridging Disciplines for Biomedicine. Proceedings of the 18th Annual International Conference of the IEEE, vol. 1. IEEE, pp. 196-197.

Raju, S., et al., 2012. Postural and ambulatory changes in regional flow and skin perfusion. Eur. J. Vasc. Endovasc. Surg. 43, 567-572.

Serov, A., et al., 2005. Full-field laser Doppler perfusion imaging and monitoring with an intelligent CMOS camera. Opt. Express 13, 3681-3689.

Simmons, G.H., et al., 2011. Changes in the control of skin blood flow with exercise training: where do cutaneous vascular adaptations fit in? Exp. Physiol. 96, 822-828.

Smit, J.M., et al., 2010. Advancements in free flap monitoring in the last decade: a critical review. Plast. Reconstr. Surg. 125, 177-185.

Svedman, C., et al., 1998. Laser Doppler imaging of skin microcirculation. Acta Derm. Venereol. Stockh. 78, 114-118.

Taylor, G.I., et al., 2011. The anatomical (angiosome) and clinical territories of cutaneous perforating arteries: development of the concept and designing safe flaps. Plast. Reconstr. Surg. 127, 1447-1459.

Tulevski, I.I., et al., 1999. Red and green laser Doppler compared with capillary microscopy to assess skin microcirculation in the feet of healthy subjects. Microvasc. Res. 58 83-88.

Tur, E., et al., 1983. Basal perfusion of the cutaneous microcirculation: measurements as a function of anatomic position. J. Invest. Dermatol. 81, 442-446.

Turner, J., et al., 2008. Current concepts in assessment of microvascular endothelial function using laser Doppler imaging and iontophoresis. Trends Cardiovasc. Med. 18, 109-116.

Wårdell, K., et al., 1993. The cutaneous vascular axon reflex in humans characterized by laser Doppler perfusion imaging. J. Physiol. 460, 185-199. 